\title{
Determination of cell proliferation
}

\author{
D M Barnes, C E Gillett
}

The proliferative activity of a tissue is a direct result of the stimulatory and inhibitory growth signals received by the cells which compose it. In cancers these messages come from activation of a number of oncogenes, probably accompanied by inactivation of one or more tumour suppressor genes and therefore knowledge of proliferative activity helps us to understand the biology of tumours. The tumour growth rate is not only a consequence of the proliferative activity but also depends on the number of cells undergoing programmed cell death (apoptosis) and necrosis. Proliferation has been studied far more extensively than apoptosis and can be used to predict prognosis and aid patient management and treatment selection. The value of proliferative activity as a prognostic factor and the methods used to measure it are still matters of debate. Several alternative methods of measurement are now available but the method of choice will depend upon the particular application required, as a marker for one tissue may not be suitable for another.

All markers identify individual cells and either measure, demonstrate or reflect a particular phase or phases of the cell cycle. The cell cycle has been well documented and primarily consists of stages $G_{1}, S, G_{2}, M$, and a resting stage $G_{0}$. In $G_{1}$ and $G_{2}$ phases cytoplasmic proteins, organelles and RNA are synthesised. In $S$ phase DNA is replicated and in $M$ phase the cell undergoes mitosis. Cells can pass from $G_{1}$ into a resting phase, $G_{0}$, from which they can re-enter the cell cycle. The time taken for the cell to complete a single cycle varies considerably but it is the $G_{1}$ phase that defines the length of the cycle. Phases $S, G_{2}$, and $M$ remain relatively constant: $S$ phase lasts between seven and 12 hours, $G_{2}$ phase between one and six hours, and mitosis from one to two hours. Therefore, fewer cells are demonstrated with a marker which detects only one of these short phases of the cell cycle.

During the cell cycle there are many checkpoints which control the progression of cells from one phase to the next and disruption of these checkpoints can lead to uncontrolled proliferation, aneuploidy and malignancy. Furthermore, under the influence of different oncogenes and tumour suppressor genes, proliferating cells can also be induced to undergo apoptosis.

Proliferation rate is a term frequently used to describe the speed at which cells are growing and reproducing in a histological section. However, the term "rate" should only apply to cellular changes which are measured over a period of time, such as when using in vivo bromodeoxyuridine (BrdU) labelling. The terms "activity" or "index" are more appropriate for describing tumour kinetics when using markers on a static cell population, such as tissue sections. Whatever method of measuring proliferation is chosen, it must fulfil a number of criteria.

\section{Requirements of the method of measurement}

Firstly, the method should be informative and relevant and, secondly, it should be reliable and reproducible. Consistent results must be obtained not only in the laboratory where the method was developed, but also in other laboratories which adopt its use. If the technique is to be applied to a large number of tissue samples it should be relatively inexpensive and, most importantly, the end result should be easy to evaluate and quantify.

Irrespective of the particular choice of method there are a number of technical problems which must be considered. The first is the type of tissue to be studied. In the past it was only possible to measure proliferation on fresh or frozen tissue. However, techniques have been adapted and antibodies are now available which can be used on fixed tissue, enabling retrospective studies to be carried out on archival paraffin wax blocks. Nevertheless, attention must still be paid to the method of fixation as delay in getting the tissues into fixative, prolonged fixation time and the type of fixative can all have profound effects on the results. ${ }^{12}$ The main problem, however, is tumour heterogeneity, the magnitude of which obviously varies between different types of tissues. Thus, care must also be taken to ensure that sampling has been adequate. When correctly carried out, fine needle aspiration samples the tissue widely, by ensuring that the tip of the needle is moved into different areas of the lesion whilst aspirating. Needle core biopsies, although containing more cells than aspirates, may not be fully representative unless the lesion has been sampled in more than one plane. The adequacy of the tissue sections depends on selection of material from the excised lesion and whether this is representative of the tissue as a whole. 
There are also problems with the method of evaluation, particularly determination of how many cells need to be counted to obtain a consistent result. For each method this varies in accordance with the proportion of labelled cells. Formulae have been derived which can be used to determine the cell count required to distinguish between two levels of proliferative activity with differing amounts of associated error. ${ }^{3}$ In tissue sections there is much debate as to which area should be selected for evaluation. Should measurements be taken at the leading edge of the tumour, presumably the most biologically active area, or does this lead to observer bias and is it better to use random selection of fields? There is also a serious danger of observer fatigue if it takes a long time to count the proliferating cells. This problem is overcome by using flow cytometry but at the expense of morphometry. Image analysis systems have also been developed to standardise evaluation but in the past these have not really been adequate; however, improvements in both hardware and software have recently been made and these systems may become of practical use in the near future.

\section{Mitotic activity index}

This is the oldest way of measuring proliferative activity. It is very cheap as mitoses can be counted in routinely fixed and processed haematoxylin and eosin sections and if performed carefully can give very useful information. The proportion of mitoses has conventionally been assessed as the number of mitotic figures per high power field, with the mean number of mitoses from 10 consecutive high power fields in the most cellular area of the tumour being measured. This method does not take into account differences in cellularity or cell size from one tumour to another. The type of fixative can affect the appearance of mitotic figures, making them look similar to pycnotic nuclei. Alcohol based fixatives not only increase the basophilia of the section as a result of protein precipitation but some can also induce considerable shrinkage of the tissue components. It has been shown at the ICRF Clinical Oncology Unit at Guy's Hospital, London, that there is a significant difference in the number of mitoses per high power field between formol saline and methacarn fixed breast cancers. Several authors have noted a reduction in the number of mitotic figures when fixation is delayed, ${ }^{145}$ although they gave different explanations for these findings. It is important to remember that delayed fixation can occur in large or very cellular specimens despite being placed in fixative within a reasonable space of time. It takes some time for the fixative to both penetrate and then fix the tissues, which can lead to inadequate fixation in the centre.

Variations in cellularity and cell size between tumours can be standardised by assessing the ratio of mitoses to the number of malignant cells. With this method the large discrepancies noted in the area of a high power field between microscopes no longer have any affect. ${ }^{6} \mathrm{De}$ monstration of mitoses is inexpensive and tech- nically easy to perform but it is important that they are accurately identified and not confused with pycnotic nuclei. Assessing the frequency of mitoses as a ratio of malignant cells can be time-consuming, although this can be reduced by using a semiquantitative method of evaluation (unpublished observations, Gillett et al, 1994). ${ }^{7}$

\section{Thymidine labelling index}

This method of assessing proliferative activity by measuring the uptake of tritiated thymidine during DNA synthesis was first introduced in the 1950s and there have been a small number of strong proponents of this technique. ${ }^{8-10}$ When used well, thymidine labelling appears to fulfil most criteria required for a satisfactory method of measurement. The technique suffers from some disadvantages: it requires small pieces of absolutely fresh tissue and depends on the successful uptake of radioactive thymidine. This uptake is demonstrated by autoradiography which is rather imprecise, while the lengthy exposure time makes the method slow. The representative nature of using and evaluating small pieces of tissue has also been questioned. ${ }^{11}$

\section{Bromodeoxyuridine labelling}

A recent improvement to the thymidine labelling technique has been the introduction of BrdU instead of thymidine. ${ }^{12}$ Antibodies are available against $\mathrm{BrdU}$, removing the need to use autoradiography. This technique has some very important advantages: again $\mathrm{BrdU}$ is incorporated into cells only during $S$ phase but this time labelling can be performed in vivo as well as in vitro. The in vitro method uses small pieces of tissue which incorporate $\mathrm{BrdU}$ under high oxygen tension once endogenous thymidine has been blocked. Tissues can then be routinely fixed and embedded in paraffin wax. This, like all other methods of measuring proliferation, addresses the proliferative state of the tissue at the moment it is removed from the body, but by labelling in vivo and then sampling the tissue within a few hours it is possible to measure the rate at which the cells are proliferating within the body. This is performed using flow cytometry to determine which phase of the cell cycle the BrdU labelled cells are in and relating this to the time between injection of BrdU and biopsy. BrdU is sometimes administered to patients as part of their treatment since it enhances the sensitivity of the tumour to radiotherapy. However, if the technique is to be used specifically for measuring proliferative activity ethical committee approval would be needed.

\section{Nucleolar organiser regions}

Nucleolar organiser regions (NORs) had a brief period of interest as a potential diagnostic or prognostic tool. The number of NORs present within the nucleolus was thought to reflect the production of ribosomal components and hence protein synthesis occurring within the 
cell. The technique was independently described in 1975 by both Goodpasture and Bloom $^{13}$ and Howell et al, ${ }^{14}$ using cell lines. A modified method for use on paraffin wax embedded material was introduced by Ploton et al in $1986 .{ }^{15}$ Thus, the main advantage of this technique was that NORs could be measured in fixed material using a simple silver solution, which at the time made it one of the few methods which could use routine, diagnostic material. ${ }^{16}$ However, some of the early proponents of the method only assessed 200 cells and some papers only referred to measuring 50 , which is a very small proportion of the entire tumour and is unlikely to be representative. Even recent publications have only evaluated 50 to 100 cells. ${ }^{1718}$ NORs are difficult to identify, time-consuming to count and do not have a consistently proven correlation with other measures of proliferative activity or prognosis.

\section{Immunohistochemical methods}

There have been several developments in this area in recent years. The first immunohistochemical method of measuring proliferation used the $\mathrm{Ki} 67$ antibody in frozen sections and cytological preparations. The Ki67 antigen is present in $G_{1}, S, G_{2}$, and $M$ phases of the cell cycle and the results obtained with this antibody fulfilled most of the criteria necessary for a proliferative marker, except that it could not be used on fixed, paraffin wax embedded material. Recently, a number of other antibodies have become available which recognise proliferation associated antigens and with technical advances, such as antigen exposure following pretreatment in a microwave oven or pressure cooker, can be used on fixed material. Monoclonal MIB $1^{19}$ and polyclonal Ki67 antibodies both recognise parts of the $\mathrm{Ki} 67$ antigen which survive the fixation process. The KiS1 antibody recognises another cell cycle associated antigen which is expressed at increasing levels during DNA synthesis and reaches a peak in mitosis, and has been shown to be prognostically useful. ${ }^{20}$ Proliferating cell nuclear antigen (PCNA) has received a lot of attention, particularly as it was one of the first antibodies which could be used on paraffin wax embedded material. However, studies have shown that PCNA has a dual role in cell replication and DNA repair. ${ }^{21}$ These two functions contribute to the conflicting data about the value of measuring proliferation with PCNA antibodies. Whilst some authors have shown it to be useful in lymphoid tissue, ${ }^{22}$ others have found it unreliable in solid tumours. ${ }^{23}$ Both KiS1 and PCNA have relatively long half lives, which means that the antigen can still be detected some time after protein production has ceased. Expression of PCNA can also be induced by growth factors in normal and benign cells which are in the vicinity of a malignant lesion. ${ }^{24}$ Hence, more cells are labelled than are actually proliferating.

Currently, there is a wealth of antibodies being developed to other cell cycle associated antigens. For most, their role in measuring proliferative activity has yet to be fully evaluated.

\section{Flow cytometry}

Since the 1960s, the flow cytometer has evolved from its basic cell counting function. Now its main roles are the measurement of both nuclear DNA and the uptake of fluorescence labelled antibodies. The assessment of ploidy and $S$ phase fraction are well documented and material from aspirates, fresh tissue and fixed, paraffin wax embedded tissue all produce valid results. ${ }^{25}$ The advantages of flow cytometry include the assessment of thousands of cells within a short space of time and in an objective manner. The disadvantages are that cell suspensions have to be made and, therefore, morphology is lost. In about one quarter of cases the DNA histograms cannot be interpreted because there are too many incomplete or poorly preserved nuclei. Furthermore, the initial cost of the machine is high. However, when flow cytometry is carried out well there is a strong correlation between the $S$ phase fraction, ploidy and prognosis. ${ }^{26}$

\section{Conclusion}

Interest in measuring proliferative activity has increased exponentially in recent years and as a consequence many new antibodies have been developed to different cell cycle associated antigens. This has been further stimulated by the ability to expose antigens by pretreatment in microwave ovens, pressure cookers or autoclaves. The ideal antigen for demonstration would be one with a short half life, which rapidly switches on during part of the cell cycle and then quickly disappears when no longer required. It can only be a matter of time before such an antigen is identified and antibodies generated against it. A possible type of candidate may be a histone protein which is synthesised at the same time as DNA. ${ }^{27}$ Probes are already available to the histone messenger RNA, enabling detection of cells in S phase using in situ hybridisation.

Despite many recent advances, inconsistencies remain with the measurement of proliferation, mainly because of differences in the accuracy of the methods of evaluation. Improved tissue preparation and automated analysis will certainly be the way forward. Already, some image analysis systems are available which, with improved programming and using artificial intelligence, can identify mitotic figures by defined characteristics with a certain degree of accuracy. Similarly, labelled nuclei, whether by antibodies, thymidine or BrdU, can be detected with image analysis but the majority of systems are still not very quick at assessing the number of cells needed to take into account the effects of heterogeneity. Currently, the most reliable, practical and useful methods of measuring proliferative activity in histological material are by counting either the fraction of mitoses or MIB1/Ki67 labelled nuclei. Although both have their disadvantages, these 
methods have been widely shown to provide useful prognostic information. ${ }^{28-32}$

1 Cross SS, Start RD, Smith JHF. Does delay in fixation affect the number of mitotic figures in processed tissue? f Clin Pathol 1990;43:597-9.

2 Cooper LC, Egan MK, Gillett CE, Springall RJ, Veldhuize $M$, Vermeeren Y. Fixation for modern histopathological techniques: an evaluation of different fixation regimes [abstract]. F Pathol 1992;167:137A.

3 Aherne WA, Camplejohn RS, Wright NA. Chapter 3. An introduction to cell population kinetics. London: Edward Arnold, 1977:17-26.

4 Graem N, Helweg-Larson K. Mitotic activity and delay in fixation of tumour tissue. Acta Pathol Microbiol Scand $(A)$ 1979;87:375-8.

5 Donhuijsen K, Schmidt U, Hirche H, van Beuningen D, Budach V. Changes in mitotic rate and cell cycle fractions caused by delayed fixation. Hum Pathol 1990;21:709-14

6 Ellis PSJ, Whitehead R. Mitosis counting - a need for reappraisal. Hum Pathol 1981;12:3-4.

7 Simpson JF, Dutt PL, Page DL. Expression of mitoses per thousand cell and cell density in breast carcinomas: a proposal. Hum Pathol 1992;23:608-11.

8 Meyer JS, Connor RE. In vitro labeling of solid tissues with tritiated thymidine for autoradiographic detection of Stritiated thymidine for autoradiographic detection

9 Tubiani M, Pejovic MH, Chavaudra N, Contesso G, Malaise EP. The long term prognostic significance of the thymidine labelling index in breast cancer. $B r f$ Cancer 1984;33:441-5.

10 Silvestrini D, Daidone MG, Valagussa P, Di Fronzo G Mezzanotte G, Bonadonna G. Cell kinetics as a prognostic indicator in node-negative breast cancer. Eur $\mathcal{F}$ Cancer Clin Oncol 1989;25:1165-71.

11 Lambert $M$. Tritiated thymidine labelling in vitro for human cancer of the breast: counting error and sampling error. Eur 7 Cancer Clin Oncol 1986;22:781-5.

12 Meyer JS, Nauert, J, Koehms S, Hughes J. Cell kinetics of human tumors by in vitro Bromodeoxyuridine labeling. $f$ Histochem Cytochem 1989;37:1449-54.

13 Goodpasture C, Bloom SE. Visualisation of nucleolar organizer regions in mammalian chromosomes using silve taining. Chromosoma 1975;53:37-50.

14 Howell WM, Denton TE, Diamond JR. Differential staining of the satellite regions of human acrocentric chromosomes. Experientia 1975;31:260-2.

15 Ploton D, Menager M, Jeannesson P, Himber G, Pigeon F, Adnet $\mathrm{J}$. Improvement in the staining and in the visualization of the argyrophilic proteins of the Nucleolar Organiser Region at the optical level. Histochem $\mathcal{F} 1986$; 18:5-14.

16 Crocker J, Skilbeck N. Nucleolar organiser region associated proteins in cutaneous melanotic lesions: a quantitative study. $\mathcal{F}$ Clin Pathol 1987;40:885-9.
17 Toikkanen S, Joensuu H. AgNOR counts have no prognostic value in breast cancer. F Pathol 1993;169:251-4.

18 Sacks NPM, Robertson JFR, Ellis IO, Nicholson RI, Crocker J, Blamey RW. Silver-staining nucleolar organiser region counts are of no prognostic value in primary breast cancer. Eur $₹$ Surg Oncol 1992;18:98-102.

19 Gerdes J, Becker MHG, Key G, Cattoretti G. Immunohistological detection of tumour growth fraction (Ki67 antigen) in formalin fixed and routinely processed tissues. $\mathcal{F}$ Pathol 1992;168:85-6.

20 Sampson SA, Kreipe H, Gillett CE, Smith P, Chaudary MA, Khan A, et al. KiS1 - a novel monoclonal antibody which recognizes proliferating cells: evaluation of its relationship to prognosis in mammary carcinoma. $f$ Pathol 1992;168:179-85.

21 McCormick D, Hall PA. The complexities of proliferating cell nuclear antigen. Histopathology 1992;21:591-4.

22 Hall PA, Levison DA, Woods AL, Yu CC, Kellock DB, Watkins JA, et al. Proliferating cell nuclear antigen (PCNA) immunolocalization in paraffin sections: an index of cell proliferation with evidence of deregulated expression in some neoplasms. $\mathcal{F}$ Pathol 1990;162:285-94.

23 Hall PA, Hart I, Goodlad R, Coates PJ, Lane DP. Expression of proliferating cell nuclear antigen noncycling cells [abstract]. F Pathol 1992;168(Suppl):97A.

24 Gillett CE, Barnes DM, Camplejohn RS. Comparison of three cell cycle associated antigens as markers of proliferative activity and prognosis in breast carcinoma. $f$ Clin Pathol 1993;46:1126-8.

25 Hedley DW, Friedlander ML, Taylor IW, Rugg CA, Musgrove A. Method for analysis of cellular DNA content of paraffin-embedded pathological material using flow of paraffin-embedded pathological material using
cytometry. $\mathcal{F}$ Histochem Cytochem 1983;31:1333-5.

26 Camplejohn RS, Barnes DM, Ash K, et al. A single centre study, in a group of 881 patients, of the prognostic significance of DNA flow cytometry. Br $\mathcal{F}$ Cancer 1995; in press.

27 Chou MY, Chang AL, McBride J, Donoff B, Gallagher GT, Wong DT. A rapid method to determine proliferation patterns of normal and malionant tissues by $\mathrm{H} 3 \mathrm{mRNA}$ in situ hybridization. Am 7 Pathol 1990;136:729-33.

28 Baak JPA, van Dop H, Kurver PHJ, Hermans J. The value of morphometry to classic prognosticators in breast cancer. Cancer 1985;56:374-82.

29 Clayton F. Pathologic correlates of survival in 378 lymph node-negative infiltrating ductal breast carcinomas. Mitotic count is the best single predictor. Cancer 1991;68: 1309-17.

30 Locker AP, Birrell K, Bell JA, Nicholson RI, Elston CW, Blamey RW, et al. Ki67 immunoreactivity in breast carcinoma: relationships to prognostic variables and shor term survival. Eur f Surg Oncol 1992;18:224-9.

31 Brown DC, Gatter KC. Monoclonal antibody Ki67: its use in histopathology. Histopathology 1990;17:489-503.

32 Pinder SE, Wencyk P, Sibbering DM, Blamey RW, Elston $\mathrm{CW}$, Ellis IO. Automated grading of breast cancer [abstract]? $\mathcal{F}$ Pathol 1994;172:21A. 